\title{
Black Holes Radiate Mainly on the Brane
}

\author{
Roberto Emparan, ${ }^{1}$ Gary T. Horowitz, ${ }^{2}$ and Robert C. Myers ${ }^{3}$ \\ ${ }^{1}$ Departamento de Física Teórica, Universidad del País Vasco, Apartado 644, E-48080 Bilbao, Spain \\ ${ }^{2}$ Physics Department, University of California, Santa Barbara, California 93106 \\ ${ }^{3}$ Department of Physics, McGill University, Montréal, Quebec, H3A 2T8, Canada
}

(Received 20 March 2000)

\begin{abstract}
We examine the evaporation of a small black hole on a brane in a world with large extra dimensions. Since the masses of many Kaluza-Klein modes are much smaller than the Hawking temperature of the black hole, it has been claimed that most of the energy is radiated into these modes. We show that this is incorrect. Most of the energy goes into the modes on the brane. This raises the possibility of observing Hawking radiation in future high energy colliders if there are large extra dimensions.
\end{abstract}

PACS numbers: 04.70.Dy, 11.10.Kk

It has been proposed that space may have extra compact dimensions as large as a millimeter [1]. If all the standard model fields live on a three-brane and only gravity (and perhaps some other unobserved fields) propagate in the bulk, such large extra dimensions are consistent with all current observations. We will consider the evaporation of black holes in this scenario. Although our results hold for any number of large extra dimensions, for definiteness we focus mainly on the case of two extra dimensions of size $L$. Since the effective four-dimensional Newton's constant $G_{4}$ is related to $G_{6}$ by $G_{4}=G_{6} / L^{2}$, if the fundamental scale of gravity in the bulk is of order a TeV, $G_{4}$ has the observed value provided $L \sim 1 \mathrm{~mm}$. For weak fields, the bulk metric can be decomposed into the fourdimensional graviton and an infinite tower of Kaluza-Klein modes, which act like four-dimensional spin-two fields with masses starting at $1 / L \sim 10^{-4} \mathrm{eV}$.

One of the most striking consequences of a low fundamental Planck scale is the possibility of forming semiclassical black holes at rather low energies, say of order $100 \mathrm{TeV}$. Suppose one collapses matter (or collides particles) on the brane to form a black hole of size $\ell_{\text {fun }} \ll$ $r_{0} \ll L$ (where $\ell_{\text {fun }}=G_{6}^{1 / 4}$ is the fundamental, i.e., sixdimensional, Planck length). This black hole has a temperature $T \sim 1 / r_{0}$ which is much larger than the mass of the light Kaluza-Klein modes. Since gravity couples to everything, and there are so many Kaluza-Klein modes with mass less than the Hawking temperature, it has been claimed $[2,3]$ that the Hawking radiation will be dominated by these Kaluza-Klein modes, with only a tiny fraction of the energy going into standard model particles. In other words, most of the energy would be radiated off of the brane into the bulk. If this were the case, the Hawking radiation from these small black holes would be essentially unobservable.

We claim that this argument is incorrect, and most of the Hawking radiation goes into the standard model fields on the brane. The easiest way to see this is to consider the calculation from the six-dimensional perspective [4]. For a single massless six-dimensional field, the rate at which energy is radiated is of order

$$
\frac{d E}{d t} \sim A_{6} T^{6} \sim \frac{r_{0}^{4}}{r_{0}^{6}} \sim \frac{1}{r_{0}^{2}},
$$

where $A_{6}$ denotes the area of the six-dimensional black hole. For a single massless four-dimensional field on the brane, the rate of energy loss is of order

$$
\frac{d E}{d t} \sim A_{4} T^{4} \sim \frac{r_{0}^{2}}{r_{0}^{4}} \sim \frac{1}{r_{0}^{2}},
$$

and hence is the same. That is, with a single relevant scale $r_{0}$ determining the Hawking radiation, bulk and brane fields must both have $d E / d t \sim r_{0}^{-2}$. Hence the Hawking evaporation must emit comparable amounts of energy into the bulk and brane. However, with the typical assumption that there are many more fields on the brane than in the bulk, one would conclude that most of the energy goes into the observable four-dimensional fields. While the detection of this Hawking radiation would likely not be the first experimental signature of large extra dimensions, such measurements would provide a dramatic new window on black hole microphysics.

We will examine this argument in more detail below (and confirm its validity), but first we must ask what was wrong with the original arguments suggesting that the Hawking radiation goes mostly into Kaluza-Klein modes. In one form [2], one views the emission of Hawking radiation as a six-dimensional process. In this case, since brane fields seem to have a tiny phase space compared to bulk fields, it would appear that the emission of the latter should dominate the Hawking evaporation. However, it is incorrect to think of brane fields as bulk fields confined to a limited phase space. The brane fields are intrinsically four dimensional, and their emission is governed by the four-dimensional relation (2), and not the six-dimensional formula (1) with a restricted area.

Dominance of the Kaluza-Klein modes might also be argued from a four-dimensional point of view [3]. In this case, it may appear that the Kaluza-Klein modes must dominate the evaporation since there are a large number [of 
$\left.\operatorname{order}\left(L / r_{0}\right)^{2}\right]$ of light modes with masses below the scale of the Hawking temperature. However, here it is incorrect to think of the individual Kaluza-Klein modes of the bulk graviton as massive spin-two fields on the brane with standard (minimal) gravitational couplings. Rather, since the Kaluza-Klein modes are excitations in the full transverse space, their overlap with the small (six-dimensional) black holes is suppressed by the geometric factor $\left(r_{0} / L\right)^{2}$ relative to the brane fields. Hence this geometric suppression precisely compensates for the enormous number of modes, and the total contribution of all Kaluza-Klein modes is only the same order as that from a single brane field. Since Eq. (1) automatically incorporates the emission of all Kaluza-Klein modes, clearly this four-dimensional approach is a complicated reorganization of a simple sixdimensional situation.

We now want to look in more detail at the rate of energy loss by a black hole to modes on the brane and in the bulk. We will consider a general dimension $d$ for the bulk spacetime, and assume that we live on a $(3+1)$ dimensional brane. The extra dimensions will have size $L$. Since we are assuming the size of the black hole $r_{0}$ is much less than $L$, the geometry near the black hole is simply that of a $d$-dimensional Schwarzschild solution,

$$
d s^{2}=-f(r) d t^{2}+f^{-1}(r) d r^{2}+r^{2} d \Omega_{d-2}^{2},
$$

with $f(r)=1-\left(r_{0} / r\right)^{d-3}$. The event horizon is at $r=$ $r_{0}$, and has area $A_{d}=r_{0}^{d-2} \Omega_{d-2}$ where $\Omega_{n}$ denotes the volume of a unit $n$ sphere.

If a black hole is formed from matter on the brane, symmetry requires that the brane pass through the equator of the black hole. We further assume that the three-brane has negligible self-gravity of its own [6]. Then the induced metric on the brane will be

$$
d s^{2}=-f(r) d t^{2}+f^{-1}(r) d r^{2}+r^{2} d \Omega_{2}^{2},
$$

with $f(r)$ still given as above. On the brane, then, the event horizon is again at $r=r_{0}$, and its area is $A_{4}=4 \pi r_{0}^{2}$. This induced metric on the brane is certainly not the fourdimensional Schwarzschild geometry. Since the Ricci tensor of this four-dimensional metric (4) is nonzero near the horizon, one can think of it as a black hole with matter fields (i.e., Kaluza-Klein modes) around it. However, the calculation of Hawking evaporation relies mainly on properties of the horizon, such as its surface gravity. Changing the geometry outside will change the effective potential that waves have to propagate through. This will modify the gray body factors, but since the potential is qualitatively the same, the total energy radiated is changed only by factors of order unity. Since the Hawking temperature is constant over the horizon, it is the same for both the black hole in the bulk and on the brane, and is given by $T=(d-3) /\left(4 \pi r_{0}\right)$.

The metric (4) (with $f$ of the form given above) has no $1 / r$ term and hence seems to give zero mass in four dimensions. However, this metric describes only the geometry near the black hole. For $r \gg L$ the geometry will be approximated by (4) with $f(r) \simeq 1-\left(2 G_{4} M / r\right)$ where $M$ is the mass of the $d$-dimensional black hole,

$$
M=\frac{(d-2) r_{0}^{d-3} \Omega_{d-2}}{16 \pi G_{d}} .
$$

In other words, the mass measured on the brane is the same as the mass in the bulk. This can be seen as follows: Consider the higher dimensional spacetime and unwrap the compact dimensions. The result is a cubic array of black holes, each of mass $M$ and separated by a distance $L$. From a large distance [7], this looks like a "surface density" $\rho=$ $M / L^{d-4}$. The asymptotic metric will thus contain the term $f(r)=1-\left(2 G_{d} \rho / r\right)$. However, since $G_{d}=G_{4} L^{d-4}$, this is equivalent to $f(r)=1-\left(2 G_{4} M / r\right)$. Although this $1 / r$ term is the dominant correction to the flat metric for $r \gg L$, it is already quite small for $r \sim L$ and will not cause a significant modification to our estimates of the energy radiated.

We now show that the emission rate of Kaluza-Klein modes, regarded as four-dimensional fields, is actually suppressed relative to modes that propagate only along the brane. In order to see this, let us consider the calculation of the emission rate of a massless bulk field in the following way: since we have to sum over all the modes of the field that are emitted by the black hole, let us decompose these according to the momentum $\mathbf{k}$ which they carry into the $d-4$ transverse dimensions. On the brane, this KaluzaKlein momentum is identified with the four-dimensional mass of these modes, which we denote $m=|\mathbf{k}|$. If we then sum over all other quantum numbers, we will find the emission rate corresponding to a Kaluza-Klein mode with momentum $\mathbf{k}$. Proceeding in this way we get, for the emission rate per unit frequency interval of modes with momenta in the interval $(\mathbf{k}, \mathbf{k}+d \mathbf{k})$,

$$
\frac{d E}{d \omega d t}(\omega, \mathbf{k}) \simeq\left(\omega^{2}-m^{2}\right) \frac{\omega A_{d}}{e^{\beta \omega}-1} d^{d-4} k .
$$

Here, $A_{d}$ is the area of the black hole in the $d$-dimensional bulk. (The only difference above for a fermionic mode would be to change the sign of the "one" in the denominator.) We are neglecting purely numerical factors since we will find below that they do not play any significant role. As a check, when this expression is integrated over all Kaluza-Klein modes, one recovers the emission rate of a massless bosonic field into the $d$-dimensional bulk:

$$
\frac{d E}{d \omega d t}(\omega)=\int_{|\mathbf{k}|=0}^{|\mathbf{k}|=\omega} \frac{d E}{d \omega d t}(\omega, \mathbf{k}) d^{d-4} k \simeq \frac{\omega^{d-1} A_{d}}{e^{\beta \omega}-1} .
$$

Consider a light Kaluza-Klein mode, with a mass much smaller than the black hole temperature, $m \ll 1 / r_{0}$. We set $d^{d-4} k \sim(1 / L)^{d-4}$ for an individual mode, and $A_{d} \sim$ $r_{0}^{d-4} A_{b}$, with $A_{b}$ the sectional area on the brane. Then,

$$
\frac{d E}{d \omega d t}(\omega, m) \simeq\left(\frac{r_{0}}{L}\right)^{d-4}\left(\omega^{2}-m^{2}\right) \frac{\omega A_{b}}{e^{\beta \omega}-1},
$$


which is identical to the emission rate of a massive field in four dimensions, except for a suppression factor of $\left(r_{0} / L\right)^{d-4}$. (Note that this formula applies equally well for $m=0$.) So we see that the Hawking radiation into each Kaluza-Klein mode (among these, the massless graviton) is much smaller than the radiation into any other minimally coupled field that propagates only in four dimensions. In particular, compared with a purely four-dimensional gravity theory, Hawking radiation in gravitons on the brane is suppressed by a factor of $\left(r_{0} / L\right)^{d-4}$. Still the total radiation (7) into a bulk field is comparable to that into a field on the brane, because there are of order $\left(L / r_{0}\right)^{d-4}$ light modes with $m<T \sim 1 / r_{0}$. As we mentioned earlier, this suppression factor can be understood as arising from the small geometric overlap between a bulk mode and a small black hole which has only a limited extent in the transverse dimensions. Of course, since there is no analogous effect for all the nongravitational fields on the brane, this supports our conclusion that most of the energy is radiated on the brane.

Since the number of relevant fields on the brane may be only a factor of 10 or so larger than the number of bulk fields, one might worry that the claim that the Hawking radiation is dominated by brane fields could still be thwarted by large numerical factors coming from the higher dimensional calculation. To check this, we consider two improvements over the rough estimate of the radiation rates given in (1) and (2). The first is to include the dimension dependent Stefan-Boltzman constant $\sigma_{n}$. In $n$ dimensions, the energy radiated by a blackbody of temperature $T$ and surface area $A_{n}$ is

$$
\frac{d E_{n}}{d t}=\sigma_{n} A_{n} T^{n} .
$$

Standard statistical mechanics calculations yield

$$
\sigma_{n}=\frac{\Omega_{n-3}}{(2 \pi)^{n-1}(n-2)} \Gamma(n) \zeta(n),
$$

with $\zeta(n)$ denoting the Riemann zeta function. These factors do not change much with the dimension, in the cases of interest. For example, $\sigma_{4}=\pi^{2} / 120 \simeq 0.08, \sigma_{6}=$ $\pi^{3} / 504 \simeq 0.06, \sigma_{10}=\pi^{5} / 3168 \simeq 0.097$. Although formally these quantities have been calculated for infinite (uncompactified) spacetimes, Eq. (9) provides a good approximation when $T \gg 1 / L$. The fact that $\sigma_{n}$ changes very little with dimension confirms that even though higher dimensional spacetimes have infinitely many more modes (corresponding to excitations in the extra dimensions), the rate at which energy is radiated by a blackbody with radius $r_{0}$ and temperature $T \sim 1 / r_{0}$ is roughly independent of the dimension.

Substituting Eq. (10) [as well as $T=(d-3) / 4 \pi r_{0}$ ] into Eq. (9), we find for the black hole that

$$
\frac{d E_{n}}{d t}=\frac{\Omega_{n-3} \Gamma(n) \zeta(n)}{(2 \pi)^{n-1}(n-2)} \Omega_{n-2}\left(\frac{d-3}{4 \pi}\right)^{n} \frac{1}{r_{0}^{2}},
$$

where we have used the horizon area for $A_{n}$. Now if we substitute in $d=6$ and take the ratio, we find $\left(d E_{4} / d t\right) /$ $\left(d E_{6} / d t\right)=56 / 5=11.2$. Hence by these calculations the emission of a bulk mode is actually suppressed relative to a mode confined to the brane. If we consider $n=d=$ 10 , the ratio becomes $\left(d E_{4} / d t\right) /\left(d E_{10} / d t\right) \simeq 12.1$.

However, there is a second improvement which we can easily incorporate into our calculations. This concerns the area that appears in (9). We have been using the horizon area as the area of the blackbody emitter in Eq. (9), but at least in the geometric optics approximation, a black hole acts as a perfect absorber of a slightly larger radius. Recall that in four dimensions there is a critical radius $r_{c}=(3 \sqrt{3} / 2) r_{0} \simeq 2.6 r_{0}$ for null geodesics. If a photon travels inside this radius, it is captured by the black hole. Detailed calculations have shown [8] that the total energy radiated is better approximated by assuming the area is given by $r_{c}$ rather than $r_{0}$. (Note that this approximation is not obviously justified since the typical wavelengths are of order the black hole size.)

We expect a similar improvement occurs in higher dimensions. For a general dimension, $r_{c}$ becomes

$$
r_{c}=\left(\frac{d-1}{2}\right)^{1 /(d-3)} \sqrt{\frac{d-1}{d-3}} r_{0} .
$$

The ratio decreases slightly with the dimension: at $d=6$, $r_{c} \simeq 1.75 r_{0} ;$ at $d=10, r_{c} \simeq 1.41 r_{0}$. This critical radius is the same for brane and bulk modes since calculating null geodesics involves only motion in a plane of the full geometry (3). This effect modifies the emission rate (11) through the area factor. Since the bulk modes include a higher power of the radius, increasing the radius increases the relative decay rates for the bulk modes by a factor $\left(r_{c} / r_{0}\right)^{n-2}$. With this correction, $\left(d E_{4} / d t\right) /\left(d E_{6} / d t\right) \simeq$ 3.66 , and $\left(d E_{4} / d t\right) /\left(d E_{10} / d t\right) \simeq 1.54$, and so the ratios become closer to one.

Thus there are no unexpected large factors to ruin the naive estimate that a Hawking evaporation emits as much energy into a typical brane field as into a typical bulk field. A definitive comparison of the bulk and brane radiation rates would require a detailed analysis. In particular, one expects a suppression for higher spin fields due to angular momentum barriers [9]. For example, in a pure fourdimensional calculation, the radiation rate for the graviton is approximately 10 times smaller than that for a massless spin-one-half field [9]. Of course, such detailed calculations would require a specific brane-world model to determine the exact black hole geometry and the precise multiplicity of bulk and brane fields.

So far we have considered small black holes with $r_{0}<$ $L$. Will larger black holes also radiate mainly on the brane? If $r_{0}>L$, the solution is simply a product of fourdimensional Schwarzschild and a torus. Hence the horizon area is $A_{d}=4 \pi r_{0}^{2} L^{d-4}$, and the geometric suppression factor in Eq. (8) is replaced by 1 . However, the Hawking temperature is now lower than the mass of all Kaluza-Klein 
modes, so their contribution to the Hawking radiation is clearly suppressed. Approximating the radiation rate with Eq. (1), we have

$$
\frac{d E}{d t} \sim A_{d} T^{d} \sim r_{0}^{2} T^{4}(L T)^{d-4} \sim\left(L / r_{0}\right)^{d-4} r_{0}^{2} T^{4} .
$$

So the total contribution of the Kaluza-Klein modes is suppressed by the factor $\left(L / r_{0}\right)^{d-4}$ relative to that of a single brane field. Actually, since $T<1 / L$, this six-dimensional formula only accurately captures the contributions of modes with relatively large Kaluza-Klein momentum. The dominant contribution will actually come from the massless mode which in this regime radiates identically to a brane field. So for large black holes a bulk field still carries essentially the same energy as a field on the brane, and the latter again dominate the Hawking radiation due to the relatively high multiplicity of light brane fields.

If a black hole initially has $r_{0}>L$, then Hawking radiation will cause the Schwarzschild radius to decrease. When $r_{0} \sim L$, the four-dimensional black hole $\times\left(S^{1}\right)^{d-4}$ solution becomes unstable [10], and is believed to break up into $d$-dimensional black holes [11]. These black holes attract each other and coalesce, forming a single higher dimensional black hole. Could this final black hole lie in the bulk and not on the brane? This is highly unlikely since a black hole will not slide off a brane. Rather it feels a restoring force due to the brane tension. To see this, we must consider the condition for a black hole on a brane to be static. A black hole will grow whenever $T_{\mu \nu} \ell^{\mu} \ell^{\nu}>0$, where $\ell^{\mu}$ is a null geodesic generator of the event horizon. This is just the statement that energy is crossing the horizon. The stress energy tensor of a brane is proportional to its induced metric. In order for the black hole to be static (and not swallow up the brane) $\ell^{\mu}$ must lie entirely in the brane so $T_{\mu \nu} \ell^{\mu} \ell^{\nu} \propto \ell_{\mu} \ell^{\mu}=0$. This will be the case if the radial direction orthogonal to the black hole is tangent to the brane. In other words, the brane must intersect the black hole orthogonally. So if one pulls on a black hole on a brane, the brane bends to stay orthogonal and pulls back on the black hole. Thus, a black hole on the brane will attract a black hole in the bulk, forming a larger black hole on the brane.

Although we have found that most of the radiation goes into purely four-dimensional fields, the evaporation of a small black hole will not proceed as in a purely four-dimensional theory. The black hole is $d$ dimensional, and its mass $M$ is related to the radius as in (5). In particular, this means that the lifetime of the black hole will not be like that of a four-dimensional black hole, $\tau_{4} \sim G_{4}^{2} M^{3}$, but rather, $\tau_{d} \sim G_{d}^{2 /(d-3)} M^{(d-1) /(d-3)}$ [2]. Note that $\tau_{d} \sim\left(L / r_{0}\right)^{2(d-4)} \tau_{4}$, and so the lifetime is longer (possibly enormously longer) than would have been expected from four-dimensional Einstein gravity. The essential feature is that when $G_{d} M<L^{d-3}$ (i.e., $r_{0}<L$ ), for a fixed mass, the Schwarzschild radius is larger than it would be for a four-dimensional black hole. This means that the temperature is lower, the horizon area is larger, and the evaporation rate is slower. The fact that the horizon area is larger is the feature which results in the higher dimensional black hole being entropically favored [10]. In the scenario with $d=6$ and $L \simeq 1 \mathrm{~mm}$, the lifetime of a black hole formed at $M \simeq 100 \mathrm{TeV}$ (so $r_{0} \sim 10^{-15} \mathrm{~mm}$ ) would be $\tau_{6} \sim 10^{-25} \mathrm{~s}$ [12]. Finally, although we have focused our discussion on the large extra dimension scenario, we have shown in [5] that black holes in the RandallSundrum scenario [13] with an infinite extra dimension (see [5,14-16]) still radiate mainly on the brane.

Given that small black holes radiate mainly on the brane (and that such black holes will not slip off the brane), the brane-world scenario has the potential to make interesting observable predictions about small black holes appearing either in collider experiments or in the early universe. It will be interesting to investigate their detailed phenomenology.

The work of R.E. is supported by UPV Grant No. 063.310-EB187/98 and CICYT AEN99-0315. G. T. H. was supported by NSF Grant No. PHY95-07065. R. C. M. was supported by NSERC of Canada and Fonds du Québec.

[1] N. Arkani-Hamed, S. Dimopoulos, and G. Dvali, Phys. Lett. B 429, 263 (1998); Phys. Rev. D 59, 086004 (1999).

[2] P. Argyres, S. Dimopoulos, and J. March-Russell, Phys. Lett. B 441, 96 (1998).

[3] T. Banks and W. Fischler, hep-th/9906038.

[4] This argument was given in a slightly different context in [5]. Similar observations were made independently by L. Susskind (private communication).

[5] R. Emparan, G. T. Horowitz, and R. C. Myers, J. High Energy Phys. 1, 7 (2000).

[6] We also assume that the brane has negligible thickness. This is reasonable since the actual thickness of the brane is likely to be of order the fundamental scale $\ell_{\text {fun }}$, and a black hole will behave semiclassically only if $r_{0} \gg \ell_{\text {fun }}$.

[7] Here, we ignore the gravitational interaction energy of the black holes in the array, which is justified for $r_{0} \ll L$.

[8] N. Sánchez, Phys. Rev. D 18, 1030 (1978).

[9] D. Page, Phys. Rev. D 13, 198 (1976); 14, 3260 (1976).

[10] R. Gregory and R. Laflamme, Phys. Rev. Lett. 70, 2837 (1993); Nucl. Phys. B428, 399 (1994).

[11] Note that at the transition with $r_{0} \sim L$, the black hole mass is $M \sim L^{d-3} / G_{d}=L / G_{4}$. Although this is much larger than the four-dimensional Planck mass, it is much smaller than a typical stellar mass (e.g., for $d=6$ and $L=1 \mathrm{~mm}$, $M=10^{27} \mathrm{~g}$, about the mass of the Earth).

[12] For a black hole with mass smaller than $10^{19} \mathrm{GeV}$, it is not meaningful to compare its lifetime with a semiclassical four-dimensional estimate.

[13] L. Randall and R. Sundrum, Phys. Rev. Lett. 83, 4690 (1999); 83, 3370 (1999).

[14] A. Chamblin, S. W. Hawking, and H. S. Reall, Phys. Rev. D 61, 065007 (2000).

[15] S. Giddings, E. Katz, and L. Randall, J. High Energy Phys. 3, 23 (2000).

[16] R. Emparan, G. T. Horowitz, and R. C. Myers, J. High Energy Phys. 1, 21 (2000). 